\title{
Research and Application of Improved Hough-ICP Algorithm
}

\author{
SU Yan \\ Guangzhou College of South China University of Technology, Guangzhou , 510800, China \\ suyan@gcu.edu.cn
}

Key words: Transportation detection; Projection wall; Hough; ICP; 3D Synthesis

Abstract: During the vehicle identification in transportation, many problems must be overcome: firstly, modern vehicle outline leads the failure of getting features from 3D rigid body. Secondly, synthesis distortion is hard to overcome in contour connecting because of front wheels torsion. So a new algorithm of 3D synthesis is presented by improved Hough and ICP analysis to solve these problems. In the stage of getting three-view drawing, Hough analysis is used to transform circle signals to line signals which are the component of newly vehicle outline, at the same time, oval signals of front wheel are fitted based on back wheel. In the contour connecting stage, a new concept named projection wall is proposed during the construction of covariance matrix based on ICP analysis, and then, the maximum values of projection walls are calculated by four-element method analysis. Finally, 3D rigid body is described accurately. Experimental results show that the proposed algorithm not only reduces the noise cased by vehicle accessories, but also overcomes signal distortion and time serious.

\section{Introduction}

At present,3D synthesis belongs to high dimensional matrix category, the popular vehicle 3D research focus on the application in the vehicle body design and auto component parts processing, including size/fluid detection, motion and strain analysis, component visual simulation ${ }^{[1]}$, but few studies have been done on the classification of vehicles. Reference [2] uses the perspective distortion modeling, and the 3D information obtained from 2D information is classified by SVM classifier; reference [3] using anisotropic magnetoresistive sensors and other auxiliary equipment, vehicles are classified by BP neural network. The rest of the "category" detection methods include electromagnetic wave detection ${ }^{[4]}$, fuzzy neural network ${ }^{[5]}$. These methods are based on multi sensor proofread 3D environment simulation, and the default function signal must be in line with the prior knowledge, which do not conform to the type of vehicle check in Guangzhou city, because Guangzhou has no prior knowledge of vehicle security, just according to the detection of the vehicle model and 3D information to verify the vehicle model and cargo capacity.

Therefore, this paper presents an improved Hough-ICP 3D synthesis algorithm based on the types of vehicle sign, which analysing outline signal through the Hough, and fitting the front wheel to get a modified three view signal, at the same time, the use of ICP analysis of the "projection wall" to achieve $3 \mathrm{D}$ contours seamlessly, and finally realize the detection of the vehicle type.

\section{Car body feature description}

\section{Car body description}

Here the paper defines: the feature that in each orthographic projection of the "three views" 
(the main view, left view, and top view) have intersection is called 3D rigid body features, and the feature that in two orthographic engineering drawing of the "three views" have intersection is called $2 \mathrm{D}$ rigid body features. As shown in Fig.1, the car 3D rigid body features mainly distributed in the edge of the chassis, front and rear bumper side edges; compared with the features of 3D rigid, 2D rigid features would be more abundant, which mainly distributed in the chassis, the roof and the front and rear bumper. The study found that 2D and 3D rigid body features can indicate the type of car only, therefore, how to overcome the weakness of the 3D rigid body features caused by the streamline shape of the modern automobile, and how to realize the seamless connection of the 3D profile of the vehicle model, is the key to get the features of the 3D rigid body. At the same time, the front wheel twist will make the 3D synthesis distortion (as shown in Fig.1 (d)) which is difficult to overcome.

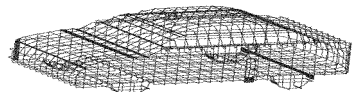

(a) 3D rigid body

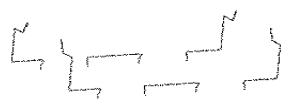

(b) $2 \mathrm{D}$ feature

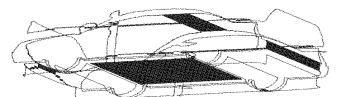

(c) $3 \mathrm{D}$ feature

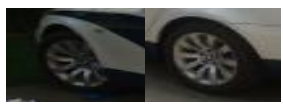

(d) Front and rear wheel contrast

Fig.1 Features of the car body

\section{Conception of projection wall}

As shown in Fig.2, the independent three view signal, because of the difference of the angle, distance, the gap and the coordinate system which is established according to the acquisition system, it leads to the mismatch between the two sides of the edge signal, or can not reach the threshold of matching, therefore, it needs to seek the maximum solution in 3D space. This paper defines: the three view signal along the $\mathrm{X}, \mathrm{Y}, \mathrm{Z}$ vertical extension, the aggregate of $3 \mathrm{D}$ signals which is surrounded by four sides is called "projection wall".

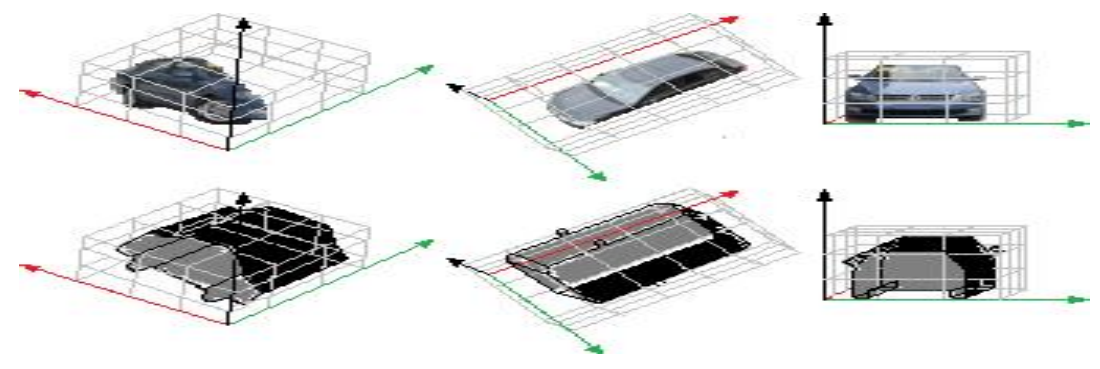

Fig.2 Concept of projection wall

\section{Improved Hough-ICP algorithm}

\section{Fitting correction}

Definition 1: suppose there is a set $\mathrm{F}$ in the $\mathrm{X}-\mathrm{Y}$ image space, there is a collection of $\mathrm{P}$ in the A-B-R parameter space. Define the set of integers $S=\left\{s_{i}\right\}, i \in R$, satisfies the formula (1), and $s_{i} \neq 0$. The $\operatorname{Sum}(\cdot)$ is a statistic which satisfies the equation.

$$
s_{i}=\operatorname{Sum}\left((x-a)^{2}+(y-b)^{2}=r^{2}\right)
$$

Definition 2: suppose there is a set $\mathrm{F}$ in the $\mathrm{X}-\mathrm{Y}$ image space, there is a collection of $\mathrm{P}$ in the A-B parameter space. Define the set of integers $S=\left\{s_{i}\right\}, i \in R$, satisfies the formula (2), and 
$s_{i} \neq 0$. The $\operatorname{Sum}(\cdot)$ is a statistic which satisfies the equation.

$$
s_{i}=\operatorname{Sum}(a x+b=y)
$$

\section{Feature extraction of projection wall}

Characteristic vector

Definition 3: suppose $F_{0} 、 F_{1} 、 F_{2}$ are respectively the left view, top view, the main view of the $2 \mathrm{D}$ vincent ${ }^{[6]}$ coefficient matrix, and meet the conditions of 1 . Its characteristic vector is defined as

$$
\begin{aligned}
& V=\left[V_{0}, V_{1}, V_{2}\right]^{T} \\
& V_{i}=\left[G\left(F_{i}\right)\right]^{T}
\end{aligned}
$$

And G(.) is the3D Vincent coefficient matrix which is extended along the direction perpendicular to the 2D plane of $\mathrm{F}_{\mathrm{i}}$.

Condition 1: suppose there is a set of integers $F=\left\{f_{1}, \cdots f_{i}\right\}, i \in R$, lets the formula $\sum_{m=-1}^{m=1} \sum_{n=-1}^{n=1} f(x+m, y+n) \leq 3$ set up. While $f(x, y)$ is the pixel value of the coordinate $(x, y)$.

\section{ICP feature points}

Definition 4: suppose there are set $\mathrm{P}$ and $\mathrm{X}$, and the set satisfies $P \cap X=N \neq \phi$.Define coordinate transformation matrix:

$$
\begin{aligned}
& q=\left[q_{R} \mid q_{T}\right] \\
& \min f(q)=\frac{1}{N} \sum_{i=0}^{N}\left\|x_{i}-R\left(q_{R}\right) p_{i}-q_{T}\right\|^{2}
\end{aligned}
$$

Where $q_{R}$ is $3 \times 3$ rotation matrix, $q_{T}$ is the unit column vector translation matrix, $\min f(q) \neq \phi$ is the minimum value of the transformation matrix $q$.

\section{Design of algorithm flow}

Step 1: vincent edge extraction of the motion blurred image signal, and eliminates non single pixel. According to the three views, the corresponding 3D coordinate system is set up, and the 3D plane signal is generated in the $X-Y-Z$ image space.

Step 2: according to formula (1) to solve the parameter space of $\left(a_{\max }, b_{\max }, r_{\max }\right)$, and the corresponding coordinates $F_{i}$ of the image space signal. Let it is the standard, rectify the front wheel which swing into the ellipse to circle. And according to the formula (2), using the same method to fit a straight line of outline signal.

Step 3: according to the formula (3) and (4) to construct the feature vector of the projection wall, according to the formula (7) to construct the covariance matrix of $F_{0} 、 F_{1}$. 


$$
\sum_{F_{0}, F_{1}}=\frac{1}{X} \sum_{i=0}^{N}\left(o_{F_{0}}-\mu_{F_{0}}\right)\left(o_{F_{1}}-\mu_{F_{1}}\right)^{T}
$$

where $o=\{x, y, z\}, \mu=\left\{\mu_{x}, \mu_{y}, \mu_{z}\right\}, \quad \mu_{x}=m_{100} / m_{000} 、 \mu_{y}=m_{010} / m_{000} 、 \mu_{z}=m_{001} / m_{000}, m_{x y z}$ can be obtained by formula (8).

$$
m_{x y z}=\frac{1}{X^{x} Y^{y} Z^{z}} \sum_{i=0}^{X} \sum_{j=0}^{Y} \sum_{k=0}^{Z} i^{x} \times j^{y} \times k^{z} \times G(i, j, k)
$$

Step 4: according to (5), the transformation matrix is constructed, and the characteristic value is calculated. The characteristic vector corresponding to the maximum characteristic value is the best rotation vector which is $q_{R}=\left[\begin{array}{llll}q_{0} & q_{1} & q_{2} & q_{3}\end{array}\right]^{T}$, and the best translation vector is $q_{T}=\mu_{F_{0}}-R\left(q_{R}\right) \mu_{F_{1}} \cdot R\left(q_{R}\right)$ can be obtained by formula (9).

$$
R\left(q_{R}\right)=\left[\begin{array}{ccc}
q_{0}^{2}+q_{1}^{2}-q_{2}^{2}-q_{3}^{2} & 2\left(q_{1} q_{2}-q_{0} q_{3}\right) & 2\left(q_{1} q_{3}+q_{0} q_{2}\right) \\
2\left(q_{1} q_{2}+q_{0} q_{3}\right) & q_{0}^{2}-q_{1}^{2}+q_{2}^{2}-q_{3}^{2} & 2\left(q_{2} q_{3}-q_{0} q_{1}\right) \\
2\left(q_{1} q_{3}-q_{0} q_{2}\right) & 2\left(q_{2} q_{3}+q_{0} q_{1}\right) & q_{0}^{2}-q_{1}^{2}-q_{2}^{2}+q_{3}^{2}
\end{array}\right]
$$

Adjust the $\mathrm{F}_{0}$ and $\mathrm{F}_{1}$ according to $q_{R}$ and $q_{T}$ to make it possible to coincide with one side of the projection wall. And according to the formula (6) to obtain the minimum value. if $\min f(q)<0.001$, then $\mathrm{F}_{0}$ and the overlap of one side of the projection wall as a benchmark, adjust the proportion $\mathrm{F}_{1}$, so that the "opposite" of the projection wall also coincide.

The same method is used to deal with the $F_{3}$, which makes the projection wall of the four sides coincide with the projection wall composed of $\mathrm{F}_{0}$ and $\mathrm{F}_{1}$, to get car body signal.

Step 5: 3D edge detection of the car body signal to extract the 3D contour signal. 2D and 3D rigid body features $E=\left\{E_{0}, E_{1}\right\}$ are separated by calculating the number of overlapping points in the $3 \mathrm{D}$ contour signal. At the same time, by using $3 \mathrm{D}$ moment invariant ${ }^{[7]}$, weighting the $\left\{E_{0}, E_{1}\right\}=\{0.15,0.85\}$, and analysis the length, width and height of the vehicle body obtained by the acquisition equipment, finally get the motorcycle type.

\section{Test and analysis}

\section{Hough fitting analysis}

Experiment 1 is to verify that the algorithm is robust to overcome the streamline signal. Fig. 3 (a), (c), (E) are three views of the original signal and curvature analysis, it can be seen that the curvature is not " 0 " is signal points with "curve", the sharp signal which number is " 2 " and " 3 " is the position that existence of edges and corners or curve trend changed significantly. Fig.3 (b), (d), (f) are the three views Hough fitting signal and curvature analysis, the contour signals of three visual icon " $\times$ " and " $\Delta$ " are linear fitting and circle fitting part based on the algorithm. It can be seen from the curvature of the corresponding analysis that in addition to the contour edges, other noise "curve" signal is eliminated, and replaced by "linear signal", so as to maximize the 
elimination of 3D rigid body characteristics caused by the lack of streamlined shape, so that it makes it possible to analyze the "overlapping" of the "projection wall" on the following steps.

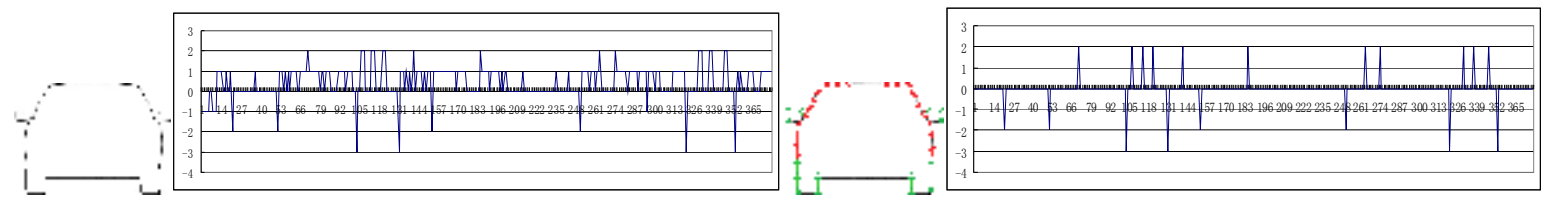

(a) Analysis of the original signal and curvature of the main view (b) Analysis of Hough fitting signal and curvature of the main view

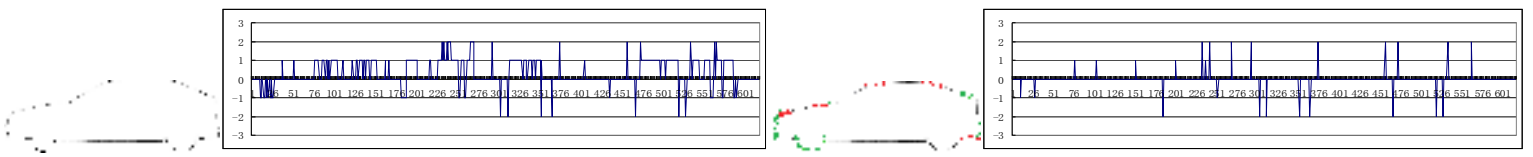

(c) Analysis of the original signal and curvature of the left view（d） Analysis of Hough fitting signal and curvature of the left view
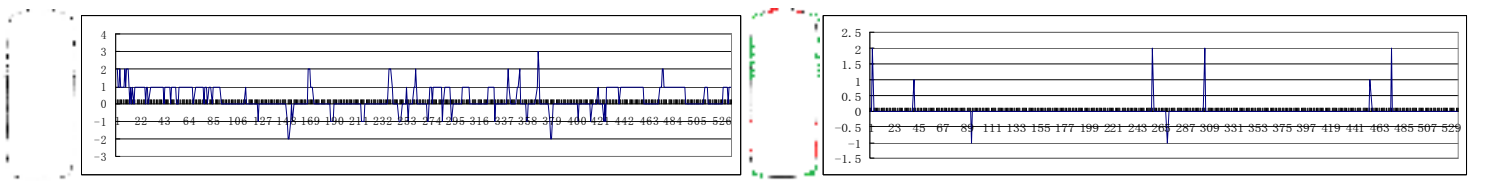

(e) Analysis of the original signal and curvature of the top view（f） Analysis of Hough fitting signal and curvature of the top view

Fig.3 Hough fitting analysis of three views

\section{Projection wall ICP synthesis}

Experiment 2 aims to present the process of ICP synthesis of the projection wall and verify its effectiveness. Fig. 4 (a) is for the three plane projection wall in a unified coordinate system according to the synthesis of the initial coordinates, the extension signal $G_{m}$ roate around the axis of $\mathrm{X}, \mathrm{Y}$ and $\mathrm{Z}$ respectively and adjust according to proportion in $3 \mathrm{D}$ space , any two projection walls in each rotation vector $q_{R}$ has a maximum overlap area. Figure 4 (b) shows overlapping pixel statistics which is get by one of the projection wall rotating around the axis of X, Y and adjusting scaling factor, it can be seen that on the same scaling factor, a pair of projection wall in a plurality of rotation angle will form a coincident extremum, so as to form a mutual connection, similar to "the back" of mountain ridge, which will have a maximum value. Fig.4 (c) is the projection drawing of Fig. 4 (b) in X, Y planes, it is intuitive to see that the maximum value is $\left(63^{\circ}, 105^{\circ}\right)$. Fig.4 (d) is the final composite image obtained by ICP analysis of the projection wall.
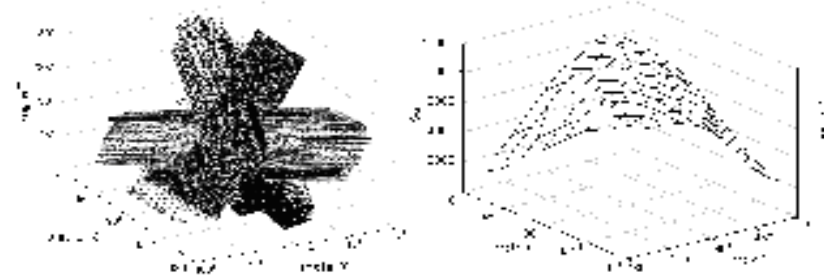

(a) Specification projection wall coordinates

(c) ICP synthetic extreme projection
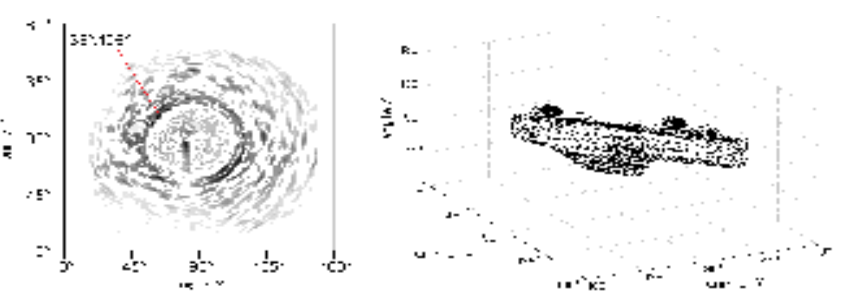

(b) ICP synthesis of extreme value statistics Fig.4 Synthesis analysis of projection wall ICP 


\section{Hough-ICP analysis}

Experiment 3 aims to verify the universality of the algorithm. Relying on the support of the Guangzhou 110 emergency center, this study obtained 17 fire cars, 55 medical cars, 65 police cars, a total of 137 experimental samples covering the fire cars, passenger, pickup ,special communication, cross-country, three car, ambulance and hatchback. The proposed algorithm is used for $3 \mathrm{D}$ synthesis and category detection. The $3 \mathrm{D}$ contour map of the second row of figure $5(\mathrm{a}) \sim(\mathrm{H})$ shows that the rigid characteristics of vehicle accurately sketched out, streamlined body characteristics are ignored, and the noise signals which influence the detection effect, such as alarm lamps, rearview mirrors, receiving antenna board of special communication are eliminated. At the same time, the third row of figure $5(\mathrm{a}) \sim(\mathrm{H})$ shows that 3D rigid features (black entity graph area in figure) distribute chassis, front and rear bumpers and side edges, while the 2D rigid features (light dashed lines) are lie scattered in vehicle body, this is the reason of weighting 2D and 3D signal $E_{0}, E_{1}$. According to the weighted analysis of $E_{0}, E_{1}$, it can lay a solid foundation for vehicle classification detection.
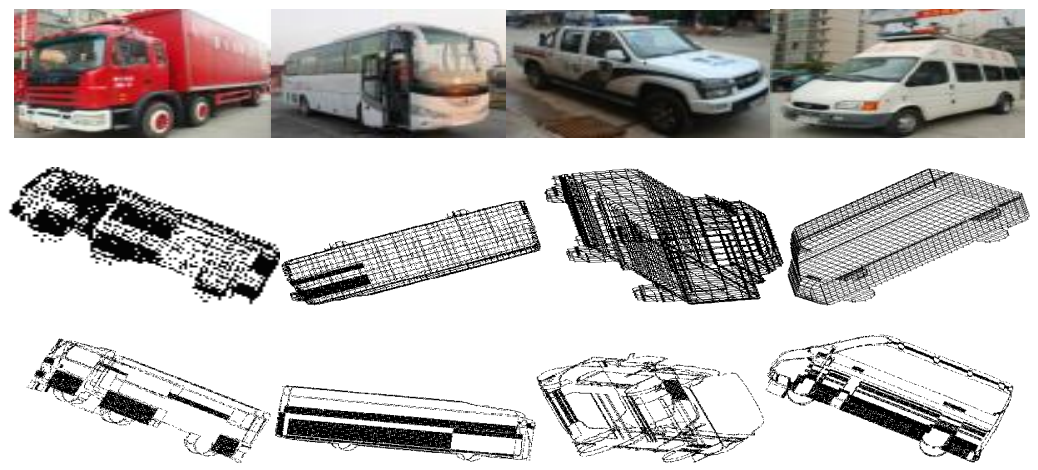

(a)Fire cars (b) Passenger（c) Pickup (d) Special communication
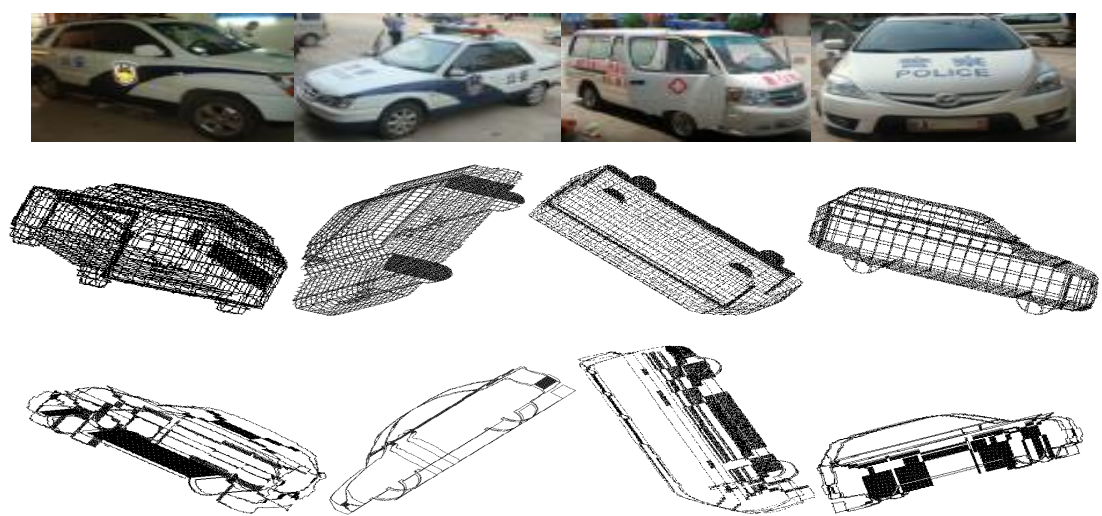

(e) Cross-country (f) Three car (g) Ambulance (h) Hatchback

Fig. 5 Vehicle detection and signals of $\mathrm{E}_{0}, \mathrm{E}_{1}$

Table 1 shows the test datas of various cars, the 3D moment invariants obtained by the proposed algorithm. The length, width, highth datas obtained by acquisition equipment parameters conversion, these 4 kinds of datas are the average of 137 samples and covers the maximum and minimum value, from this comprehensive analysis can match the corresponding model. It can be seen from the table that the difference of the sample of the fire engine is bigger, so its parameter is floating big, the other car models are stable in the small interval. 
Table1 Vehicle inspection dates

\begin{tabular}{|c|c|c|c|c|}
\hline Parameters & 3D moment invariants & length $(\mathrm{m})$ & width $(\mathrm{m})$ & highth $(\mathrm{m})$ \\
\hline Far types & $23.54782 \pm 0.24286$ & $9.73 \pm 2.33$ & $3.21 \pm 0.53$ & $3.26 \pm 0.42$ \\
\hline Passenger & $21.02467 \pm 0.01473$ & $9.22 \pm 0.73$ & $3.10 \pm 0.23$ & $3.10 \pm 0.31$ \\
\hline Pickup & $9.12687 \pm 0.01224$ & $4.11 \pm 0.30$ & $1.30 \pm 0.21$ & $1.30 \pm 0.20$ \\
\hline Special communication & $12.95186 \pm 0.01695$ & $4.20 \pm 0.24$ & $1.44 \pm 0.21$ & $1.92 \pm 0.20$ \\
\hline Cross-country & $11.34568 \pm 0.01138$ & $4.12 \pm 0.31$ & $1.54 \pm 0.12$ & $1.31 \pm 0.22$ \\
\hline Three car & $10.71439 \pm 0.01436$ & $4.33 \pm 0.10$ & $1.42 \pm 0.20$ & $1.21 \pm 0.21$ \\
\hline Ambulance & $13.75169 \pm 0.02130$ & $4.20 \pm 0.21$ & $1.42 \pm 0.31$ & $1.69 \pm 0.20$ \\
\hline Hatchback & $10.11245 \pm 0.01716$ & $4.20 \pm 0.10$ & $1.41 \pm 0.20$ & $1.21 \pm 0.21$ \\
\hline
\end{tabular}

\section{Conclusion}

This paper has the characteristics of foresight and exploration, the proposed improved Hough-ICP algorithm for 3D synthesis can detect vehicle type. Aim at 3D rigid body features missing and contour synthesis distortion which caused by streamlined body and the twist of the front wheel, the algorithm through Hough analysis outline signals with streamlined feature, and use the after wheel as the basic circle to fitting front wheel; using the ICP analysis "projection wall" to find the extremum to achieve 3D contour profile seamlessly, finally achieve the purpose of detecting vehicle type. It is worth mentioning that the design of the algorithm without any other auxiliary software and special hardware, but the speed up of the algorithm depends on the robustness of the bearing system.

\section{Acknowledgements}

This work was financially supported by the Guangdong Provincial Youth Innovative Talents Project (2015KQNCX231) and the first 2014 collaborative education center project in Guangdong province (SC141201)

\section{References}

[1] Ge Guangying. Vehicle detection and recognition algorithm based on SVM [J]. Computer Engineering, 2006,33 (6): 6-11.

[2] Chen Hong, He Xiaohai. Application of SVM to the recognition of 3D vehicle model [J]. Journal of Sichuan University (Natural Science Edition), 2006,43 (6): 1279-1284.

[3] Yang Bo, Ni Wen Bin. Vehicle detection and vehicle classification based on anisotropic magnetoresistive sensor [J].Journal of scientific instrument, 2013,33 (3): 537-544.

[4] LANJH, XIANGY, WANGLP, et al. Vehicle detection and classification by measuring and processing magnetic signal [J].Measurement, 2011, 44(1), 174-180. 
[5] ZHANGJL, XUH, LIUJ N, et al. PID control based on fuzzy neural net work for precision angular alignment [J]. Journal of Scientific Instrument, 2012, 33(3): 549-554.

[6] Mao Xianguang. Study on transverse refers to the recognition system and the key technology

[D]. Guangzhou: South China University of Technology, 2010

[7] Qin Xujia, Wang Jianqi. Point cloud splicing of 3D invariant moment feature [J]. Journal of mechanical engineering, 2013,49 (1): 129-134. 